\title{
Measurements and monitorings dependent color intensity of drugs and food supplements interactions with textile dyes between TLC plates by using rope printing technique
}

\begin{abstract}
Pharmacovigilance between drug and food supplements is common in the world and it is important to take precautions before clinical cases. In this study, interactions between food supplements containing herbal food mixes, sweeteners, vitamin blends and commonly used painkillers, antibiotics and anticoagulants, were revealed with helping of macrame ropes, smartphone, TLC plates and textile dyes and color analysis method supported image j program. The sensitivity of the developed method was high (LOD: 0.7ppm, LOQ: 2ppm). Color analysis results can provide a color risk scale that can be combined with online drug interaction softwares such as Micromedex.
\end{abstract}

Keywords: drug-food interaction, textile dyes, rope printing, color intensity analysis, TLC plates
Volume 7 Issue 5 - 2019

\author{
Ozan Emre Eyupoglu \\ Biochemistry Department, School of Pharmacy, Istanbul Medipol \\ University, Turkey
}

\begin{abstract}
Correspondence: Ozan Emre Eyupoglu, Biochemistry Department, School of Pharmacy, Istanbul Medipol University, Kavackk South Campus, Goztepe Dist. Ataturk Str. No.40, 348I0, Beykoz-Istanbul/Turkey, Tel +90538 58820 70, Email oeeyupoglu@medipol.edu.tr
\end{abstract}

Received: September II, 2019 | Published: October 08, 2019

\section{Introduction}

The discussions of pharmacokinetic and pharmacodynamic interactions between drugs and medicinal plants has been put forward, especially with medicinal herbs that can interact with antidiabetic drugs. In addition to their treatment, many diabetic patients are known to use herbal medicines that have both antidiabetic effects and potential benefits. ${ }^{1}$ Approximately $72.8 \%$ of people with diabetes use herbal medicine and dietary supplements. ${ }^{2}$ Interactions between herbal mixtures and medicines may increase the effectiveness of antidiabetic agents. For example, antidiabetic drugs have been shown to increase blood glucose-lowering effects with agrimony. ${ }^{3}$ Many anti-diabetic drugs are substrates of the CYP450 enzyme system, and many medicinal plants may also affect this system. For example, ginkgo inhibits CYP3A4, CYP2C9 and CYP2C19, while St John's wort inhibits CYP2C and CYP3A. ${ }^{4}$ Some of the commonly used antidiabetic drugs include pancreatic beta-cell receptors, $\alpha$-glucosidase inhibitors, e.g., acarbose, peroxisome proliferator activated receptor activators, e.g., thiazolidindiones. ${ }^{5}$ Many of these plants which include bio-active molecules such as peptides, alkaloids, lipids, terpenoids, amines, sulphur compounds, coumarins, steroids, flavonoids, and inorganic ions, have been used in traditional medicine as antidiabetics. ${ }^{6}$ As the use of herbal medicines increases, short-term or long-term toxicity due to side effects, overdose, hypersensitivity can be detected by pharmacodynamics or pharmacovigilance. ${ }^{7}$ In 2010, as a good example practice, the Upsala monitoring center created a database of 4 million reports on about approximately 21000 herbal products from 100 countries in the World. ? In the US, herbal products are classified as botanicals or dietary supplements, not medicines. In Europe, the definition of a herbal product as a food or medicine may have a significant effect on pharmacovigilance, as there is no legal requirement for food supplements. ${ }^{7}$ Classifications of adverse reactions of herbal medicines in orthodox medicine were formed as Type A (acute); dose-dependent, Type B (specific); not dose-dependent, Type C (chronic): cumulative effect, Type D (onset); genotoxic, carcinogenic. ${ }^{8}$

Adverse effects reports were generally about unaware of the using of herbal and nutritional products by physician or patient. ${ }^{9}$ There have been an extensive list of herbal remedies used in hospital environments in Thailand. ${ }^{10}$ Consumers and industries can report adverse events related to the use of dietary supplements via the electronic forms MedWatch 3500, 3500A and 3500B, an adapted safety reporting portal by FDA. ${ }^{11}$ For example, it has been possible that mixtures of pharmaceuticals containing aristolochic acid from Chinese and Western pharmaceutical components may cause kidney damage, and mixtures of drugs containing tripterygium glycosides may cause reproductive system disorders and mixtures of Polygonum multiflorum from the buckwheat family may cause liver damage. ${ }^{12}$ Unexpected adverse effects from digitalis, garlic, senna extract, mustard oil, menthol, aloe, and turmeric plant mixtures have been reported. ${ }^{13}$ With comparative pharmacoepidemiology, prospective, meta-analysis, retrospective studies, case control and cohort studies, the safety of herbal medicines has been investigated by testing the signals detected from the self-reporting program systems. ${ }^{14}$ The scope of drug-herbal food studies should be extended to include drug use review, photochemical analysis, prescription sequence symmetric analysis, quality monitoring, quantification of components and nonclinical safety studies. ${ }^{15}$ Organic anion carriers containing more than 10 transmembrane transport proteins have great potential in many dietary supplements-drugs, plant-endogenous compounds, drugdrugs, plant-drug interactions. Organic anion carriers play an important 
role in the excretion and detoxification of many water-soluble drugs, compounds or additives (eg: nonsteroidal anti-inflammatory drugs, neurotransmitter metabolites, mycotoxins, phenolic acids and flavonoids) from the kidneys and liver, respectively. ${ }^{16}$ Both in vitro and in vivo studies have shown that some flavonoids may have the ability to regulate organic anion transporter (OAT) activity (eg, Apigenin inhibits OAT1 activity while catechin inhibits OAT4). Flavonoids present in most plants may cause plant-drug interactions in combination with antiviral and antibiotics. ${ }^{17}$ The natural sweetener stevioside and its aglycone metabolite steviol reduce the renal clearance of some anionic drugs as they show a high affinity for both OAT1 and OAT3. ${ }^{18}$

The use of multiple drugs and dietary supplements is very common, especially in AIDS and cancer patients. ${ }^{19,20}$ A mixture of dextromethorphan, midazolam (oral and intravenous administration), tolbutamide and caffeine were administered to healthy people for evaluation of intestinal and hepatic activities by using a cocktail approach and a greater effect was observed in the small intestine than the liver. ${ }^{21}$ Consumption of large amounts of grapefruit juice, apple juice or orange juice reduces plasma levels of fexofenadine (antihistamine), an organic anion carrier substrate in the intestine, by inhibition according to in vitro evaluations. ${ }^{22}$

The British Drug Safety Committee reported five cases of warfarin anticoagulation interacting with cranberry juice. ${ }^{23}$ Red yeast rice, obtained by fermenting Monascus purpureus yeast on rice, and was developed as a dietary supplement to lower blood lipids, could increase the dosage of verapamil. ${ }^{24}$ Pharmacists and health professionals should educate patients appropriately to minimize possible adverse juice-drug interactions. ${ }^{25}$

Standardised questionnaires were used to determine risky uses of herbal medicines and dietary supplements in cancer treatment. ${ }^{26}$ More than half of the reported 108 herb supplement-drug interaction cases were sourced of green tea $(13.9 \%)$, garlic $(14.8 \%)$, ginger $(3.7 \%)$, mistletoe $(9.3 \%)$, Chinese herbs $(8.3 \%)$. The most commonly reported drugs that interact with herbal supplements were cyclophosphamide, irinotecan, warfarin, nonsteroidal antiinflammatory drugs, paclitaxel and vinorelbine. For example, garlic, combined with aspirin and omeprazole, increased the risk of gastrointestinal bleeding. The interactions between green tea supplements and irinotecan, warfarin or cyclophosphamide were also possible. ${ }^{27}$ Catechin and caffeine compounds in green tea inhibited the release of arachidonic acid from platelets and inhibited thromboxane and clot formation. Dietary supplements such as Marshmallow, Barley, Iceland Moss, Rice Bran, Coffee Charcoal, Quince could reduce the absorption of warfarin. ${ }^{28}$ Mania induction was observed in depressive patients who mixed panax ginseng and antidepressants, while Chinese herbal product, xaio chai hu tang (sho-saiko-to), caused a decrease in blood concentrations together with prednisolone. Gummy soluble fibers could reduce the absorption of drugs. ${ }^{29}$ In a cell culture experiment, Caco- 2 cell monolayers containing hepatocytes were used to investigate the invitro effects of various components (curcumin, hesperetin, quercetin, naringenin, and piperine) in irinotecan transport and the study was followed by in vivo. ${ }^{30}$ Potential interaction of warfarin with vitamin E, coenzyme Q, ginkgo, ginseng, papaya, garlic, Devil's clam, Danshen, Dong quai, fish oils increased international normalized ratio, while potential interaction of warfarin with St. John's wort and green tea decreased international normalized ratio. ${ }^{31}$ apixaban and edoxaban), whose safety profile is superior to vitamin $\mathrm{K}$ antagonists, which are more interacted with herbal supplements or foods, have begun to replace warfarin. ${ }^{32}$ Some of the anti-retroviral agents used for the treatment of HIV (eg; ritonavir, nevirapine, saquinavir, efavirez) inhibit CYP3A4 enzymes and interact with warfarin, is a drug with narrow therapeutic index, to diminish its effectiveness. ${ }^{33}$ Royal jelly which is a thick, milky white food produced by bees by worker bees (Apis mellifera L.) contraindicates against acute asthma exacerbation, eczema, shortness of breath, hemorrhagic colitis, runny nose, conjunctivitis, and atopy, facial edema, can cause a possible bleeding risk by interacting with warfarin. ${ }^{34}$ Calcium-supplemented foods increase antibiotic resistance and create therapeutic failure. Malnutrition may also affect the absorption of drugs. The absorption of oral drugs by the intestine is usually in competition with nutrients. Therefore, for example, diuretics can cause side effects on urinary potassium loss as well as impaired cardiac function. ${ }^{35}$ Alcohol intake causes increased renal excretion of folate and poor absorption of niacin, vitamin $\mathrm{C}$, thiamine, vitamin $\mathrm{A}$ and vitamin $\mathrm{B}_{6}{ }^{36}$

Foods such as carbonated soft drinks, tea, chocolate, and coffee containing methylxantane may interact with medications and create deep and undesirable effects on the young central nervous system. ${ }^{37}$ The interaction between an anti-depressant and vitamin B complex intake may have a potential effect on the central nervous system. ${ }^{38}$ Curcumin increases the glutathione S-transferase activity while valerian reduces uridine diphosphoglucoronosyl transferase activity. Due to these enzymatic effects, drug plasma levels are adversely affected. ${ }^{39}$ Medications used in the last 2 weeks of life for 166 adult patients under palliative care were screened for potential interactions by using the 'Stockley's Drug Interactions On-line 10th Edition' software with clinical pharmacists. Decreasing body mass and decreasing renal and hepatic clearance for these patients may help to tolerate side effects of drugs. ${ }^{40}$ Interactions between herbal supplements and analgesic, anti-cancer drugs related to palliative care have been documented. Detected supplement-drug interactions that increase the risk of potential bleeding included cod liver oil and diclofenac, garlic and ibuprofen, cranberry juice and warfarin. ${ }^{41}$ Drug interaction software such as online Lexi-Interact and Micromedex are widely used by prescribers to identify clinically meaningful drug interactions because interactions between several drugs can be controlled at the same time. ${ }^{42}$ Despite the hepatoprotective effects of nutraceuticals such as chlorogenic acid, syringic acid, eriodictyol, caffeic acid, ferulic acid, naringenin, resveratrol, quercetin, rosmarinic acid, it has been reported that they can adversely affect the efficacy and toxicity of analgesics and antipyretic drugs, containing acetaminophen. ${ }^{43}$ Since it is important to develop different techniques to detect all these interactions before they become clinical cases, the work on this paper can be valuable. In spite of the fact that thinlayer chromatography (TLC) plates have been useful for quantitative analysis of pharmaceutical products and routine qualitative analysis of plant extracts ${ }^{44}$ the use of TLC plates in combination with the developed rope printing technique to investigate drug-herbal food supplement interactions in vitro is not available in the literature. For this purpose, in this work, the ropes dipped in different drug-food supplement liquid mixtures were dyed with textile dyes and then printed between TLC plates and the power of interaction (synergist and antagonist side effects) was rated by measuring the color intensity of these prints with helping of Image $\mathrm{J}$, a color analysis program (Figure 1).

Oral anticoagulants, containing Factor Xa inhibitors (rivaroxaban, 


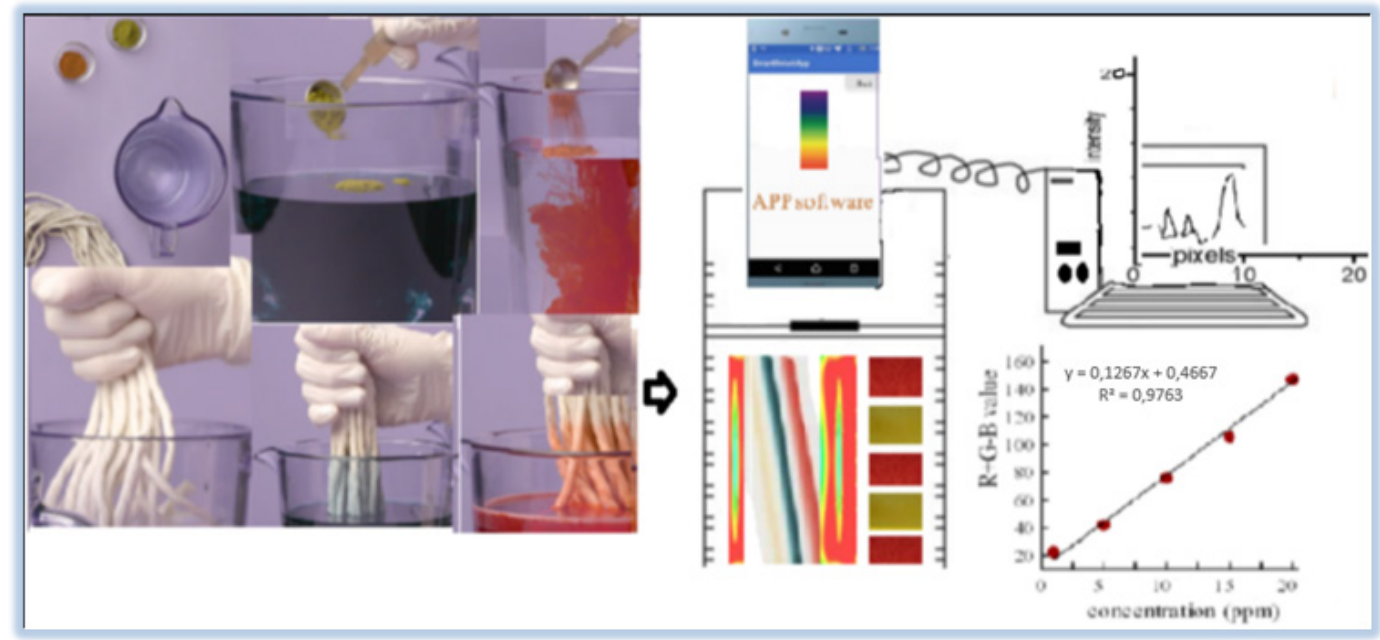

Figure I Color-analysis flow diagram of drug-food supplement interaction after rope staining and TLC printing.

\section{Materials and methods}

All textile dye reagents were solved with ultrapure water produced by using Milli-Q ${ }^{\circledR}$ IQ 7003/7005 Ultrapure Lab Water System (Merck). Dylon trademark Green-Fabric Dye and Tulip Red Fabric Dye were purchased from Migros shopping market, Turkey and prepared by adding to $150 \mathrm{~mL}$ mixtures of hot ultrapure water and table salt, separately for rope dyeing. Green Chain vegetable powder (135 g, 30 sashe), contains 46 kinds of herb mixes such as Ginkgo biloba, green tea, garlic extracts and more, was obtained from The LifeCo Company, Turkey. A solution of $4.5 \mathrm{~g}$ of a powder mixture was prepared by stirring with IsoLab magnetic mixturer in $150 \mathrm{~mL}$ of warm water. Orzax Ocean Multi Vitamin Mineral Fish Oil Syrup (food supplement flavored with honey and orange aroma, $150 \mathrm{~mL}$ ), Supradyn All Day (30 Tablet) vitamin and mineral supplement, Aspirin ${ }^{\circledR}$ (Acetylsalicylic acid) $100 \mathrm{mg}$, (20 Tablet), AKSEF $500 \mathrm{mg}$ (cefuroxime axetil) (10 tablet) and Coumadin (Warfarin Sodium) 10 mg, (28 tablet) drugs were purchased from pharmacy, Turkey for used as interaction samples. A solution of one tablet from each drugs in $150 \mathrm{~mL}$ of warm water was prepared separately. TLC Silica gel 60 $\mathrm{F}_{254}$ Aluminium sheets $(20 \times 20 \mathrm{~cm})$ were purchased from OrLab ${ }^{\circledR}$ laboratory market in order to create the printing surface. YarnArt $50 \mathrm{~g}$ beige cotton macrame rope $(5 \mathrm{~mm}$ thick and single auger) was purchased from haberdashery, Turkey to be used in dyeing and printing. With an imaging system consisting of an Apple iPhone 8 64GB smartphone webcam and a multivariate image analysis program (Image J), print images between TLC plates reflecting the intensity of interaction after dyeing with textile dyes of ropes dipped in different drug-food supplement mixtures were depicted by using as inputs of partial least squares with helping plot chart of color values for red (R), green $(\mathrm{G})$ and blue $(\mathrm{B})$ parts of the images. Red and green color rope print lines were produced between TLC plates and Images of rope print lines were photographed with helping a smartphone. The zone area lines of images were imported in software to compute the color pixel densities proportional to the severity of drug-food supplement interactions (Figure 1).

In addition, function of MATLAB with chemometric methods was used in combination of matrix values with number of pixels. While pure black or white or unused blue color areas were specified with triple matrix code " $[\mathrm{R}=\mathrm{G}=\mathrm{B}=0]$ ", the others were specified with binary matrix code " $[R=G=1]$ ". For the image color quality, $\%$ average relative prediction error was determined based on the distance between the TLC plate and the mobile phone webcam. If this error affects red and green color depth, it is indicated by -1 matrix code. ${ }^{45}$ With good precision and accuracy, high sensitivity, statistical validation parameters of this improved method which is one similar of computational quantification tests using image, ${ }^{46}$ were measured and regression curve data of this improved method were performed by using Microsoft Excel (Microsoft Office Corporation, 2010, Redmond, Washington) and SPSS Version 21.0 software program. All statistical analysis were reported significantly $(p<0.05)$ with standard deviation. The limit of detection (LOD) and limit of quantification (LOQ) were determined by the average of standard deviation of the calibration curves by created via Zoner Photo Studio 17 program in terms of 3 times and 10 times of rate of 'slope / standard deviation' obtained from the calibration graphs of color line areas of all drugfood supplement interactions respectively in which the smallest pixel color depth parameters (with CIELAB (L*, a*, b*, h ${ }^{\circ}, \mathrm{K} / \mathrm{S}$ ) color measurement method ${ }^{47}$ ) of rope printing images on TLC plates painted with textile dyes taken with the help of smartphone. Colour intensity values may be transformed into a pseudo absorbance value and then plotted against concentration to obtain linearized plots. ${ }^{48}$

\section{Results and discussions}

Detection of colorless molecules based on reversible and rapid color change is an example of molecular recognition as it is based on strong molecular structures and shapes. Cetirizine, one of the most commonly antihistamine drugs, used in the treatment of allergy, competes with phenolphthalein, a color indicator to form an inclusion complex with $\beta$-cyclodextrin. ${ }^{49}$

Visible absorption spectra of coal tar dye spots in commercial cosmetics were measured with a reversed phase TLC plate by using a scanning densitometer. ${ }^{50}$ The color density analysis of a yellowish brown complex formed by passing arsine gas through a filter paper pre-soaked in mercury bromide solution was found by scanning the image and calculating with the help of software. ${ }^{51}$ In a study, photo color analysis measurements under light source of wool fabrics dyed 
with pomegranate peel, madder and alkanet root were shown with angle and tint color parameters (CIELAB (L*: Light-dark difference, $a^{*}$ : Red-green difference, $b^{*}$ : Yellow-blue difference, $h^{\circ}$ : Light angle) and ratio of absorption and scattering coefficients $(\mathrm{K} / \mathrm{S}){ }^{52} \mathrm{In}$ an other study, for detecting the $\mathrm{SO}_{2}$ concentrations of twenty-five practical food samples, practical assay platform including paper chip combined color analysis application installed on a smartphone was developed. ${ }^{53}$ Subtle color and shape changes caused by sulfur dioxide in the air in moss leaves were detected by a simple webcam and imaging processing algorithm to observe the risks of chemical exposure through the plant. ${ }^{54}$ To quickly detect formaldehyde in food samples, a simple paper-based analytical device (PAD) was developed based on the reaction of formaldehyde with excess sulphide to form sodium hydroxide and its acid-base titration with phenolphthalein color indicator and via using of a digital camera. ${ }^{55}$

The $\lambda_{\max }$ values of microgram amounts of dyes (Victoria Blue R, methylene blue and fluorescent) were measured on TLC plates by using new sample application discs and TLC scanner. ${ }^{56}$ In this study, Calibration graphs of the color intensities of the TLC line fields in the

Table I Matrix and color depth parameters of drug-food interactions captured images were created (Figure 1). Detection limits (LOD: 0.7 ppm, LOQ: 2 ppm) were respectively determined as 3 times and 10 times of the average ratios (slope/standard deviation) obtained from the calibration graphs with triplicate smartphone applications. Linear range was found between 0.5 ppm-3 ppm (Table 1). For intra-day and inter-day conditions, the differences in color intensity measurements between the wet or dried dyed images and standard errors due to close and distant filming to images of TLC plates were indicated with a matrix value of -1 in the triple color matrix. All results were statistically significant $(p<0.05)$. Green and red color densities were expressed as +1 matrix value in linear rope print images on TLC plates (Table 1). Before identical macrame ropes were dipped into the 0.01 sashe green chain vegetable powder aqueous solution and into half tablet Aspirin ${ }^{\circledR}$ (Acetylsalicylic acid) aqueous solution separately, it was dipped into red and green fabric dye solutions and pressed between TLC plates. After identical macrame ropes were dipped into the 0.01 sashe green chain vegetable powder aqueous solution and into half tablet Aspirin ${ }^{\circledR}$ (Acetylsalicylic acid) aqueous solution separately and dried for 30 seconds separately, dyeing and pressing processes were repeated between TLC plates.

\begin{tabular}{|c|c|c|c|c|c|c|c|c|c|}
\hline \multirow[b]{2}{*}{ Sample Interactions } & \multicolumn{3}{|c|}{ Matrix color codes } & \multicolumn{6}{|c|}{ CIELAB Pixel color depth } \\
\hline & $\left(x_{1}\right)$ green & $\left(x_{2}\right)$ red & $\left(x_{3}\right)$ blue & $\mathbf{L}^{*}$ & $\mathbf{a}^{*}$ & $\mathbf{b}^{*}$ & $h^{\circ}$ & K/S & $\begin{array}{l}\text { Percentage } \\
\text { error }\end{array}$ \\
\hline Green chain powder+Aspirin & 0 & I & 0 & $61.27 \pm 0.02$ & $3.27 \pm 0.03$ & $30.42 \pm 0.04$ & $85.33 \pm 0.01$ & $|1.7| \pm 0.03$ & $0.17 \%$ \\
\hline Green chain powder+Aksef & 0 & 1 & 0 & $53.42 \pm 0.02$ & $4.35 \pm 0.03$ & $32.45 \pm 0.04$ & $83.42 \pm 0.01$ & $10.93 \pm 0.03$ & $0.30 \%$ \\
\hline Green chain powder+warfarin & 1 & 0 & 0 & $55.24 \pm 0.02$ & $5.16 \pm 0.03$ & $34.85 \pm 0.04$ & $78.65 \pm 0.01$ & $9.72 \pm 0.03$ & $0.50 \%$ \\
\hline Supradyn all day+Aspirin & 1 & -1 & 0 & $65.19 \pm 0.02$ & $2.33 \pm 0.03$ & $36.48 \pm 0.04$ & $93.75 \pm 0.01$ & $8.75 \pm 0.03$ & $0.75 \%$ \\
\hline Supradyn all day+Aksef & -1 & I & 0 & $50.42 \pm 0.02$ & $6.42 \pm 0.03$ & $33.41 \pm 0.04$ & $90.45 \pm 0.01$ & $12.33 \pm 0.03$ & $0.85 \%$ \\
\hline $\begin{array}{l}\text { Orzax Ocean MultiVitamin Mineral Fish } \\
\text { Oil Syrup+Aspirin }\end{array}$ & 1 & 1 & 0 & $47.25 \pm 0.02$ & $6.85 \pm 0.03$ & $31.33 \pm 0.04$ & $77.33 \pm 0.01$ & $6.55 \pm 0.03$ & $0.25 \%$ \\
\hline $\begin{array}{l}\text { Orzax Ocean MultiVitamin Mineral Fish } \\
\text { Oil Syrup+Aksef }\end{array}$ & 1 & 1 & -1 & $49.83 \pm 0.02$ & $2.79 \pm 0.03$ & $28.46 \pm 0.04$ & $79.45 \pm 0.01$ & $15.78 \pm 0.03$ & $1.20 \%$ \\
\hline $\begin{array}{l}\text { Orzax Ocean MultiVitamin Mineral Fish } \\
\text { Oil Syrup+warfarin }\end{array}$ & I & -1 & -1 & $51.12 \pm 0.02$ & $4.65 \pm 0.03$ & $27.12 \pm 0.04$ & $91.15 \pm 0.01$ & $\mid 7.21 \pm 0.03$ & $1.50 \%$ \\
\hline
\end{tabular}

$L^{*}$, Light-dark difference; $a^{*}$, Red-green difference; $b^{*}$,Yellow-blue difference; $h^{\circ}$, Light angle; $(K / S)$, ratio of absorption and scattering coefficients.Values are means of triplicate determinations $(p<0.05)$

Rope printing images of TLC plates were photographed by smart phone with three repetitions. Before and after the drug-food supplement interactions, the differences in color intensities of linear prints of rope printing increased depending on the forces of the interactions with color tone clarity from Image J programme (Figure 1). In contrast, red and green fabric dye solutions interacting with identical macrame ropes dipped into 0.1 tablet AKSEF (cefuroxime axetil) and 5 tablet Coumadin (warfarin sodium) aqueous solutions, separately, decreased in color intensities of linear prints of rope printing. This manner was understood from the increase in the $\mathrm{L}^{*}$ value from the pixel color depth parameters because sulfo groups in fabric dyes may interact with hydroxyl groups in warfarin and amino groups in cefuroxime axetil more than carbonyl groups in acetylsalicylic acid (Table 1). All concentrations were adjusted to a single dose $(0.0003 \mathrm{~g} / \mathrm{mL})$ for easy comparison of staining color intensities with each other. While 0.1 tablet Supradyn All Day vitamin and mineral supplement aqueous solution caused the discoloration of the rope suppression of the dyes between TLC plates by interacting with Aspirin ${ }^{\circledR}$ and Coumadin drugs, it caused darkening of color with aqueous solution of 0.1 tablet AKSEF antibiotic drug. Color lightening showed negative interaction and color darkening showed positive interaction (Table 1). Orzax Ocean multi vitamin mineral fish oil syrup was negatively interacting with all medicines (Aspirin ${ }^{\circledR}, \mathrm{AKSEF}$, and Coumadin) because the free carboxyl group of the arachidonic acid derivative in 
fish oil can interact with drugs. Color scale bandages with green and red fabric dyes were applied as indicators of drug-food supplement interactions. In the all printing process between TLC plates with dyed rope, measuring of color intensity as a blind trial against water was removed from all actual color interaction trials.

\section{Conclusion}

In Turkey, in vitro qualitative and quantitative measurements of interactions of intensely sold commercial dietary supplements and commonly used painkillers, antibiotics, each other based on the color determination were demonstrated in this paper for the first time and in this study, proven results with color analysis can be associated with commonly used online drug interaction softwares such as Lexiinteract and Micromedex for creating risk color scales inserted drug package. This in-vitro study, which is an easy and fast applicable emergency triage without clinical experience about drug-food supplement interactions also supports in-vivo studies.

\section{Ethics approval}

This article does not contain any studies with human participants or animals performed by the author.

\section{Acknowledgments}

I would like to thank my family for their moral support. I did not receive any financial funding or material supports interested with the article.

\section{Conflicts of interest}

The author stated that there is no conflict of interest relevant to this study.

\section{References}

1. Gupta RC, Chang D, Nammi S, et al. Interactions between antidiabetic drugs and herbs: an overview of mechanisms of action and clinical implications. Diabetol Metab Syndr. 2017;9(1):59.

2. Chang HY, Wallis M, Tiralongo E. Use of complementary and alternative medicine among people living with diabetes: literature review. J Adv Nurs. 2007;58(4):307-319.

3. Gray AM, Flatt PR. Actions of the traditional anti-diabetic plant, Agrimony eupatoria (agrimony): effects on hyperglycaemia, cellular glucose metabolism and insulin secretion. Br J Nutr. 1998;80(1):109-114.

4. Mills E, Montori $\mathrm{V}, \mathrm{Wu} \mathrm{P}$, et al. Interaction of St John's wort with conventional drugs: systematic review of clinical trials. $B M J$. 2004;329(7456):27-30.

5. Jovanovic L, Hassman DR, Gooch B, et al. Treatment of type 2 diabetes with a combination regimen of repaglinide plus pioglitazone. Diabetes Res Clin Pract. 2004;63(2):127-134

6. Chang CL, Lin Y, Bartolome AP, et al. Herbal therapies for type 2 diabetes mellitus: chemistry, biology, and potential application of selected plants and compounds. Evid Based Complement Altern Med. 2013;2013:378657.

7. Debbie S, Graeme L, Pierre D, et al. Pharmacovigilance of herbal medicine. J Ethnopharmacol. 2012;140(3):513-518.

8. Edwards IR, Aronson JK. Adverse drug reactions: definitions, diagnosis and management. Lancet. 2000;356(9237):1255-1259.

9. Williamson EM, Driver S, Baxter K. (Eds.), Stockley's Herbal Medicines Interactions. Pharmaceutical Press: UK; 2009. 432 p.

10. Saokaew S, Suwankesawong W, Permsuwann U, et al. Safety of herbal products in Thailand: an analysis of reports in the thai health product vigilance center database from 2000 to 2008. Drug Safety. 2011;34(4):339350 .

11. Safety Reporting Portal of the Food \& Drug Administration (FDA) and the National Institutes of Health (NIH).

12. Zhang L, Yan J, Liu X, et al. Pharmacovigilance practice and risk control of traditional Chinese medicine drugs in China: current status and future perspective. J Ethnopharmacol. 2012;140(3):519-525.

13. Kalaiselvan V, Saurabh A, Kumar R, et al. Adverse reactions to herbal products: an analysis of spontaneous reports in the database of the Pharmacovigilance Programme of India. J Herbal Med. 2015;5(1):48-54.

14. Shaw D, Graeme L, Pierre D, et al. Pharmacovigilance of herbal medicine. J Ethnopharmacol. 2012;140(3):513-518.

15. Pratt N, Chan EW, Choi NK, et al. Prescription sequence symmetry analysis: assessing risk, temporality, and consistency for adverse drug reactions across datasets in five countries. Pharmacoepidemiol Drug Saf. 2015;24(8):858-864.

16. Mor AL, Kaminski TW, Karbowska M, et al. New Insight Into Organic Anion Transporters From The Perspective of Potentially Important Interactions and Drugs Toxicity. J Physiol Pharmacol. 2018;69(3):307324.

17. An G, Wang X, Morris ME. Flavonoids are inhibitors of human organic anion transporter 1 (OAT1)-mediated transport. Drug Metab Dispos. 2014;42:1357-1366.

18. Srimaroeng C, Chatsudthipong V, Aslamkhan AG, et al. Transport of the natural sweetener stevioside and its aglycone steviol by human organic anion transporter (hOAT1; SLC22A6) and hOAT3 (SLC22A8). $J$ Pharmacol Exp Ther. 2015;313(2):621-628.

19. Richardson MA, Ramirez T, Palmer JL, et al. Complementary/alternative medicine use in a comprehensive cancer center and the implications for oncology. J Clin Oncol. 2000;18(13):2505-2514.

20. Sparber A, Wootton JC, Bauer L, et al. Use of complementary medicine by adult patients participating in HIV/AIDS clinical trials. $J$ Altern Complement Med. 2000;6(5):415-422.

21. Huang SM, Lesko LJ. Drug-Drug, Drug-Dietary Supplement, and DrugCitrus Fruit and Other Food Interactions: What Have We Learned? J Clin Pharmacol. 2004;44(6):559-569.

22. Dresser GK, Bailey DG, Leake BF, et al. Fruit juices inhibit organic anion transporting polypeptide-mediated uptake to decrease the oral availability of fexofenadine. Clin Pharmacol Ther. 2002;71(1):11-20.

23. British Committee on Safety of Medicines: Current problems in pharmacovigilance, September 2003.

24. Fung WT, Subramaniam G, Lee J, et al. Assessment of Extracts from Red Yeast Rice for Herb-Drug Interaction by in-vitro and in-vivo assays. Sci Rep. 2012;2:298.

25. Chen M, Zhou S, Fabriaga E, et al. Food-drug interactions precipitated by fruit juices other than grapefruit juice: An update review. J Food Drug Anal. 2018;26(2S):S61-S71.

26. Zeller T, Muenstedt $\mathrm{K}$, Stoll $\mathrm{C}$, et al. Potential interactions of complementary and alternative medicine with cancer therapy in outpatients with gynecological cancer in a comprehensive cancer center. J Cancer Res Clin Oncol. 2013;139(3):357-365.

27. Alsanad SM, Williamson EM, Howard RL. Cancer Patients at Risk of Herb/Food Supplement-Drug Interactions: A Systematic Review. Phytother Res. 2014;28(12):1749-1755.

28. Nutescu EA, Shapiro NL, Ibrahim S, et al. Warfarin and its interactions with foods, herbs and other dietary supplements. Expert Opin Drug Saf. 2006;5(3):433-451. 
29. Fugh-Berman A. Herb-drug interactions. Lancet. 2000;355(9198):134138

30. Bo L, Baosheng Z, Yang L, et al. Herb-drug enzyme-mediated interactions and the associated experimental methods: a review. $J$ Tradit Chin Med. 2016;36(3):392-408.

31. Greenblatt DJ, von Moltke LL. Interaction of Warfarin With Drugs, Natural Substances, and Foods. J Clin Pharmacol. 2005;45(2):127-132.

32. Di Minno A, Frigerio B, Spadarella G, et al. Old and new ora anticoagulants: Food, herbal medicines and drug interactions. Blood Rev. 2017;31(4):193-203.

33. Liedtke MD, Rathbun RC. Drug interactions with antiretrovirals and warfarin. Expert Opin Drug Saf. 2010;9(2):215-223.

34. Lee NJ, Fermo JD. Warfarin and Royal Jelly Interaction. Pharmacotherapy 2006;26(4):583-586.

35. McCabe BJ. Prevention of food-drug interactions with special emphasis on older adults. Curr Opin Clin Nutr Metab Care. 2004;7(1):21-26.

36. Light KE, Hakkak R. Alcohol and nutrition. In: McCabe BJ, Frankel EH, Wolfe JJ, editors. Handbook of food-drug interactions. Boca Raton: CRC Press; 2003. 167-189 p.

37. Appendix D.13. Caffeine content of common beverages and food. In McCabe BJ, Frankel EH, Wolfe JJ, editors. Handbook of food-drug interactions. Boca Raton: CRC Press; 2003. 517-521 p.

38. De Boer A, van Hunsel F, Bast A. Adverse food-drug interactions. Regul Toxicol Pharmacol. 2015;73(3):859-865.

39. Fasinu PS, Bouic PJ, Rosenkranz B. An overview of the evidence and mechanisms of herb-drug interactions. Front Pharmacol. 2012;3:69.

40. Morgan NA, Rowett D, Currow DC. Analysis of drug interactions at the end of life. BMJ Support Palliat Care. 2015;5(3):281-286.

41. Sparreboom A, Cox MC, Acharya MR, et al. Herbal Remedies in the United States: Potential Adverse Interactions with Anticancer agents. $J$ Clin Oncol. 2004;22(12):2489-2503.

42. Haidar C, Jeha S. Drug interactions in childhood cancer. Lancet Oncol. 2011;12(1): 92-99.

43. Bousova I, Skalova L. Inhibition and induction of glutathione S-transferases by flavonoids: possible pharmacological and toxicological consequences. Drug Metab Rev. 2012;44(4):267-286.
44. Migas P, Borys I. BMD-TLC-A Useful Separation Technique for Quantitative Analysis of Arbutin and Hydroquinone in Herbal Drugs. Acta Chromatogr. 2012;24(2):291-300.

45. Hemmateenejad B, Mobaraki N, Shakerizadeh-Shirazia F, et al. Multivariate image analysis-thin layer chromatography (MIA-TLC) for simultaneous determination of co-eluting components. Analyst. 2010;135(7):1747-1758

46. Anwar J, Waheed-uz-Zaman, Shafique MU et al. Computational Quantification of Spot Tests by Image Scanning-A New Analytical Technique for Micro Samples. Anal Lett. 2010;43(2):367-371.

47. Shinde S, Sekar N. Synthesis, spectroscopic characteristics, dyeing performance and TD-DFT study of quinolone based red emitting acid azo dyes. Dyes Pigments. 2019;168:12-27.

48. Birch NC, Stickle DF. Example of use of a desktop scanner for data acquisition in a colorimetric assay. Clin Chim Acta. 2003;333(1):95-96.

49. Khalafi L, Kashani S, Karimi J. Molecular Recognition: Detection of Colorless Compounds Based On Color Change. J Chem Educ. 2015;93(2):376-379.

50. Ohno T, Mikami E, Matsumoto H. Identification of Oil-Soluble Coal Tar Dyes in Cosmetics Using Reversed-Phase TLC/Scanning Densitometry. J Health Sci. 2003;49(5):401-404.

51. Salman M, Athar M, Waheed-uz-Zaman, et al. Micro-determination of arsenic in aqueous samples by image scanning and computational quantification. Anal Methods. 2012;4(1):242-246.

52. Yilmaz F, Bahtiyari MI. Coloration of Wool Fabrics with Various Plantal Sources. J Text Eng. 2017;24(106):62-71.

53. Fu LM, Liu CC, Yang CE, et al. A PET/Paper Chip Platform for High Resolution Sulphur Dioxide Detection in Foods. Food Chem 2019;286:316-321.

54. Qin X, Zhu Y, Yu J, et al. Chemical Sensing in Real-time with Plants Using a Webcam. Anal Chem. 2018;90(21):13030-13035.

55. Taprab N, Sameenoi Y. Rapid Screening of Formaldehyde in Food using Paper-Based Titration. Anal Chim Acta. 2019;1069:66-72.

56. Bonicamp JM, and Moll EB. Column Chromatography of Dyes: Microanalysis with a Thin Layer Chromatography Scanner Works to Beat the Bands. Microchem J. 1997;55(2):145-150. 